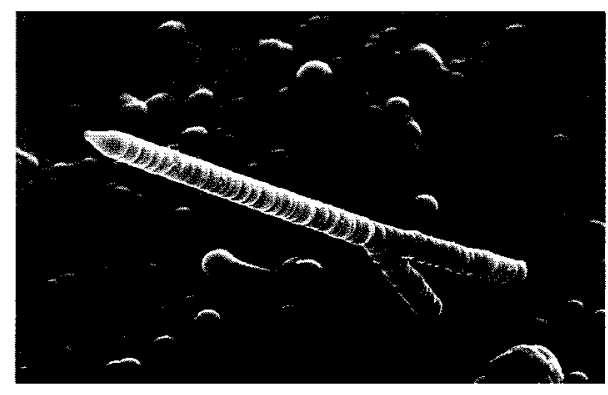

\section{7}

\section{Kohlenstoffasern aus der Gasphase}

urch Gasphasenpyrolyse von Kohlenwasserstoffen erhält man Kohlenstoffasern, die nach der Graphitierung besser geordnet sind als herkömmliche, über ein Spinndüsenverfahren erzeugte, Fasern. Sie können Längen von mehreren Zentimetern und Dicken von einigen hundert Mikrometern erreichen.

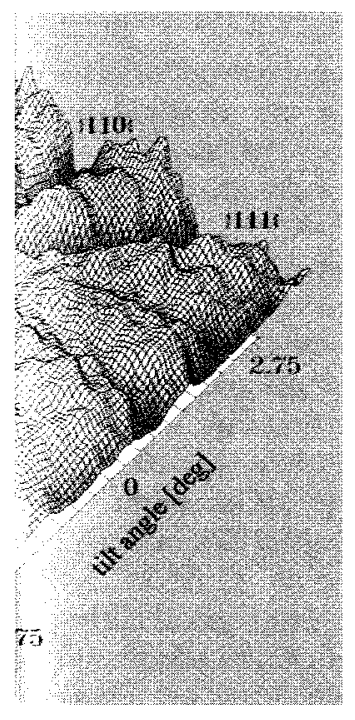

\section{1}

Spionage in der Festkörperphysik

Der Massensepaam CERN, Europas größtem Forschungszentrum für Teilchenphysik, dient zur Erzeugung radioaktiver Ionenstrahlen sehr hoher Reinheit und Intensität. Doris Forkel-Wirth berichtet über die vielfältigen Möglichkeiten, die ISOLDE für die Erforschung mikroskopischer Eigenschaften von Festkörpern mit Hilfe kernphysikalischer Methoden bietet. Man erhält sowohl Informationen über strukturelle und elektronische Eigenschaften als auch über Diffusionsprozesse.

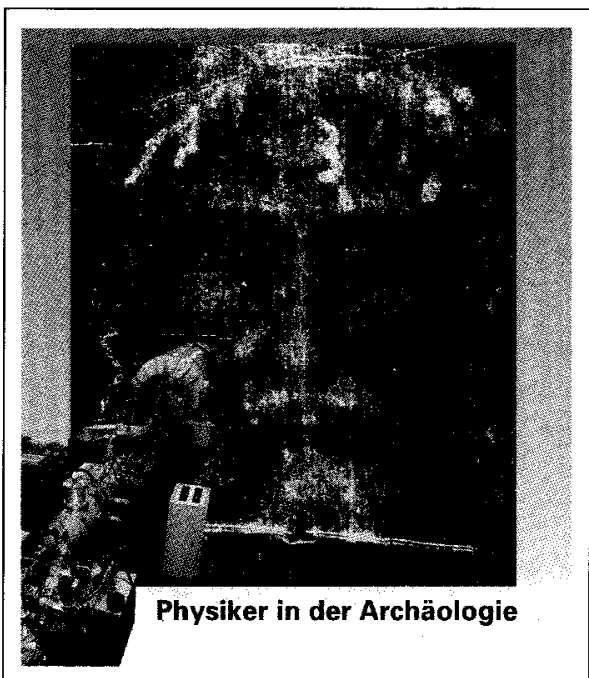

Tm Jahre 1389 erwähnt der Bischof von ITroyes in einem Brief an den damaligen Papst Klemens VII ein Leichentuch, auf dem der Abdruck eines Gekreuzigten erkennbar ist (siehe Titelbild). Rund sechshundert Jahre später bestimmen drei internationale Gruppen von Physikern mit Hilfe der Radiokarbonmethode unabhängig voneinander das Alter dieses Tuches. Dieses Verfahren, das einen wichtigen Platz in der Arbeit der Kunsthistoriker und Archäologen gefunden hat, beschreibt Willy Woelfli, einer der drei Gruppenleite auch an weiteren ausgewählten Beispielen auf den Seiten 58 bis 66 .

\section{1}

\section{Weder Fermionen noch Bosonen}

Während in drei Dimensionen nur zwei nämlich Fermionen und Bosonen, gibt es in zweidimensionalen Räumen neue Möglichkeiten, die Anyonen. Sie erfreuen sich bei theoretischen Physikern in den letzten zehn Jahren eines regen Interesses.

\section{Trtelgesehron?}

58 Archäologie mit einem Schwerionenbeschleuniger Willy Woelfli Standardisierung und Kalibrierung von ${ }^{14} \mathrm{C}$-Daten

\section{Aus der roned w.}

67 Hubble ist wohlauf Fullerene im Handel Supraleitung bei 250 Kelvin? Ultraschnelles photochromes Material

\section{Festhorporou}

71 „Spionage“ in der Festkörperphysik Doris Forkel-Wirth

\section{Spielwites:}

79 Der Flug des geflügelten Samens Hans-Joachim Schlichting,

Christian Ucke

\section{Quanten (n)}

81 Weder Fermionen noch Bosonen Gert-Ludwig Ingold

Zwei harmonisch gekoppelte Anyonen Komplexe Zahlen

\section{Matertamsson.}

87 Kohlenstoffasern aus der Gasphase Jürgen Walter

Graphitierung von Kohlenstoffen

\section{Software}

93 Btoher

95 Im narebron the
Kuratorium

G. Blatter, Zürich

Gerhard Fritsch, München

Thomas Grandke, Erlangen

Ute Gummich, Frankfurt

Eckhardt Hoenig, Jena

Friedemar Kuchar, Leoben
Hansheinrich Langhoff, Würzburg

Heinrich Mitter, Graz

Hans Jürgen Schmitt, Aachen

Werner Schneider Erlangen

Peter Schuster, Jena

Pedro Waloschek, Hamburg
Redaktion

Thomas Bührke, Weinheim

Bernhard Kramer, Hamburg

Grafik

Klaus Marinoff, Leimen

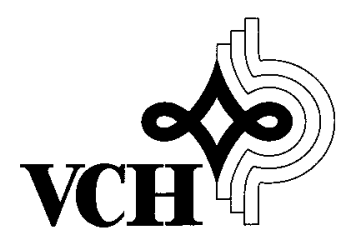




\title{
Weder Fermionen noch Bosonen
}

\author{
Gert-Ludwig Ingold
}

\begin{abstract}
Während in drei Dimensionen nur zwei Arten von Teilchen existieren können, nämlich Fermionen und Bosonen, gibt es in zweidimensionalen Räumen neue Möglichkeiten, die Anyonen.
\end{abstract}

A lle physikalischen Teilchen lassen sich in Azwei Gruppen einteilen, die Fermionen und die Bosonen. Zu ersteren, die nach dem italienischen Physiker Enrico Fermi benannt wurden, gehören insbesondere die Bausteine der Materie, nämlich das Proton, das Elektron und das Neutron. Diese Teilchen sind dadurch ausgezeichnet, daß sie halbzahligen Spin tragen. Die Bosonen, für die der indische Physiker Satyanendranath Bose Pate stand, tragen dagegen immer ganzzahligen Spin. Zu ihnen gehören zum Beispiel die Pi-Mesonen mit Spin 0 und das Photon mit Spin 1.

Mit dem Spin ist eine andere, sehr wichtige Eigenschaft eng verknüpft. Bei der Vertauschung zweier Teilchen kommt es darauf an, ob es sich um Fermionen oder Bosonen handelt, wie wir später noch genauer sehen werden. Dies hat weitreichende Konsequenzen. So gäbe es beispielsweise kein Periodensystem der Elemente, wenn Elektronen nicht Fermionen wären. Andererseits ist es nur möglich, Laser zu bauen, weil Photonen Bosonen sind.

Seit einiger Zeit ist bekannt, daß eine Beschränkung des Raumes auf zwei Dimensionen dazu führt, daß nicht mehr nur noch Bosonen und Fermionen möglich sind. Man spricht dann allgemein von Anyonen (die nicht mit Anionen, also negativ geladenen Ionen, verwechselt werden sollten). Diese englische Namensgebung geht auf den amerikanischen Physiker Franz Wilczek zurück und bedeutet soviel wie irgendwelche Teilchen, also etwas allgemeineres als Fermionen und Bosonen. Natürlich leben reale physikalische Teilchen immer in drei Dimensionen, wo weiterhin nur die altbekannten Fermionen und Bosonen vorkommen können. Es gibt jedoch
1
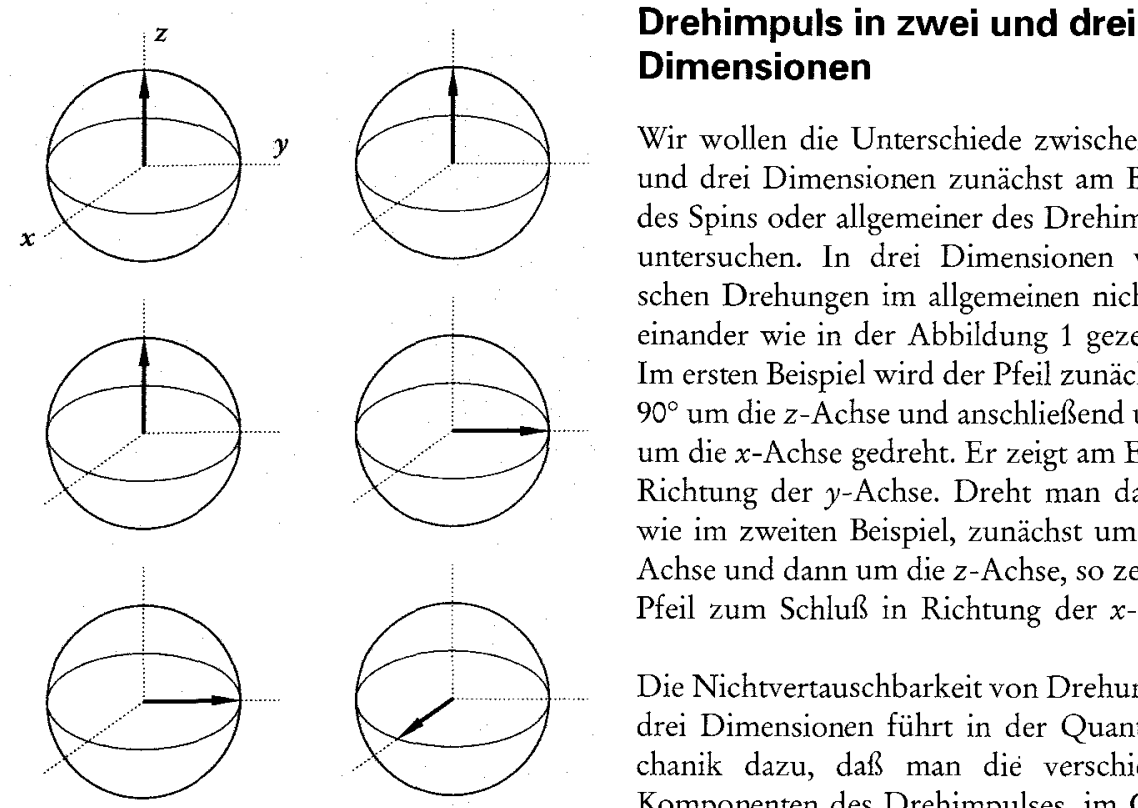

Abb. 1. Nichtvertauschbarkeit von Drehungen in drei Dimensionen. Links wird zunächst um die $z$-Achse und anschließend um die $x$-Achse gedreht. Rechts ist die Reihenfolge umgekehrt, und es ergibt sich eine andere Endstellung des Pfeils.

physikalische Systeme, die näherungsweise als zweidimensional angesehen werden können, bei denen also die Ausdehnung in der dritten Raumrichtung vernachlässigt werden kann. Da in solchen Systemen Zustände denkbar sind, die sich wie Anyonen verhalten, ist es durchaus sinnvoll, die Physik in zwei Dimensionen genauer zu untersuchen. Entsprechend erfreuen sich Anyonen in den letzten zehn Jahren vor allem unter theoretischen Physikern regen Interesses.

\section{Drehimpuls in zwei und drei}

Wir wollen die Unterschiede zwischen zwei und drei Dimensionen zunächst am Beispiel des Spins oder allgemeiner des Drehimpulses untersuchen. In drei Dimensionen vertauschen Drehungen im allgemeinen nicht miteinander wie in der Abbildung 1 gezeigt ist. Im ersten Beispiel wird der Pfeil zunächst um $90^{\circ}$ um die $z$-Achse und anschließend um $90^{\circ}$ um die $x$-Achse gedreht. Er zeigt am Ende in Richtung der $y$-Achse. Dreht man dagegen, wie im zweiten Beispiel, zunächst um die $x$ Achse und dann um die $z$-Achse, so zeigt der Pfeil zum Schluß in Richtung der $x$-Achse.

Die Nichtvertauschbarkeit von Drehungen in drei Dimensionen führt in der Quantenmechanik dazu, daß man die verschiedenen Komponenten des Drehimpulses, im Gegensatz zur klassischen Mechanik, nicht gleichzeitig scharf messen kann. Eine weitere und für uns hier wichtigere Konsequenz, deren Beweis allerdings genauere Kenntnisse der Quantenmechanik und etwas Rechnung erfordert, besagt, daß Drehimpulse nur halboder ganzzahlige Werte annehmen können.

Ganz anders ist die Situation in zwei Dimensionen. Hier sind nur noch Drehungen um eine Achse, beispielsweise die $z$-Achse möglich. Führt man zwei Drehungen hintereinander aus, so ergibt sich insgesamt eine Drehung um die Summe der beiden Winkel. Es ist also völlig unerheblich, in welcher Reihenfolge die beiden Drehungen ausgeführt werden. Diese neue Situation führt dazu, daß in zwei Dimensionen die Drehimpulse nicht mehr auf halb- oder ganzzahlige Werte festgelegt sind. 


\section{Vertauschung von Teilchen in zwei und drei Dimensionen}

Wir haben schon erwähnt, daß sich Bosonen und Fermionen verschieden verhalten, wenn man sie miteinander vertauscht. Zur genaueren Untersuchung der Vertauschung von zwei Teilchen betrachten wir zunächst zwei Tischtennisbälle. Um diese zu unterscheiden, können wir sie verschieden farbig anmalen, zum Beispiel schwarz und weiß. Legen wir die Tischtennisbälle nebeneinander, so können wir natürlich unterscheiden, ob der linke Ball der schwarze oder der weiße ist, und wir können somit sofort feststellen, ob jemand die beiden Bälle miteinander vertauscht.

Die Situation ändert sich jedoch, wenn wir stattdessen beispielsweise Elektronen betrachten. Diese können wir nicht anmalen oder in irgendeiner anderen Weise markieren, sie sind also ununterscheidbar. Dieses wichtige Prinzip bedeutet, daß sich das Ergebnis einer Messung an einem System von Elektronen nicht ändern darf, wenn man die Elektronen untereinander vertauscht. Diese Ununterscheidbarkeit läßt in drei Dimensionen nur zwei Klassen von Teilchen zu: Bosonen und Fermionen.

Um dies zu verstehen, müssen wir uns etwas genauer mit der quantenmechanischen Beschreibung von Teilchen beschäftigen. Während man den Zustand eines klassischen Teilchens durch dessen Ort und Geschwindigkeit charakterisieren kann, ist dies in der Quantenmechanik nicht mehr möglich. Im allgemeinen befindet sich nämlich ein quantenmechanisches Teilchen nicht an einem genau definierten Ort. Man kann vielmehr für jeden Ort $x$ nur eine Wahrscheinlichkeit $p(x)$ angeben, das Teilchen dort $z u$ finden. Die Ortsaufenthaltswahrscheinlichkeit $p(x)=$ $|\psi(x)|^{2}$ ist das Betragsquadrat einer komplexen Funktion, der sogenannten Wellenfunktion $\psi(x)$, die den Zustand des Teilchens vollständig beschreibt. Die Wellenfunktion selbst läßt sich nicht messen, wohl aber die Ortsaufenthaltswahrscheinlichkeit. Wir haben daher die Freiheit, die Wellenfunktion mit einem Phasenfaktor $e^{i \varphi}$ zu multiplizieren, ohne daß sich an Meßergebnissen etwas ändert.

Wir haben nun die nötigen Voraussetzungen, um die Vertauschung zweier ununterscheidbarer, quantenmechanischer Teilchen zu diskutieren. Die beiden Teilchen seien durch die Wellenfunktion $\psi\left(x_{1}, x_{2}\right)$ beschrieben, wobei sich die erste Variable immer auf das erste Teilchen und die zweite Variable auf das zwei-
Abb. 2. Vertauschung von Teilchen in zwei Dimensionen. Unter den beiden letzten Zuständen ist der jeweilige Phasenfaktor der Wellenfunktion relativ zum Ausgangszustand angegeben.

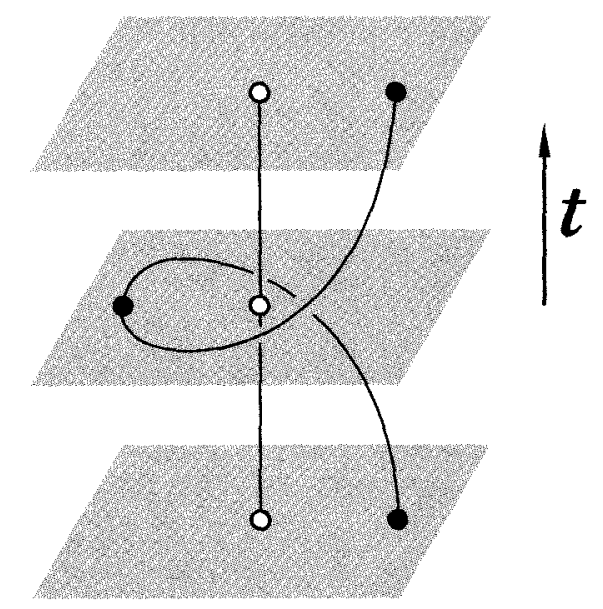

Abb. 3. Weltlinien zweier Teilchen, die in zwei Dimensionen umeinander herumgeführt werden.

te Teilchen beziehen soll. Wir haben also in Gedanken die Teilchen numeriert, wohlwissen, daß diese Numerierung keine physikalische Relevanz haben darf. Vertauschen wir die beiden Teilchen, so wird aus $\psi\left(x_{1}, x_{2}\right)$ die neue Wellenfunktion $\psi\left(x_{2}, x_{1}\right)$. Dabei muß jedoch $\left|\psi\left(x_{1}, x_{2}\right)\right|^{2}=\left|\psi\left(x_{2}, x_{1}\right)\right|^{2}$ sein, da wir sonst die beiden Fälle anhand der meßbaren Ortsaufenthaltswahrscheinlichkeit unterscheiden könnten. $\psi\left(x_{1}, x_{2}\right)$ und $\psi\left(x_{2}\right.$, $x_{1}$ ) dürfen sich also nur um einen Phasenfaktor unterscheiden. Bei einer weiteren Vertauschung erhalten wir nochmals denselben Phasenfaktor, sind aber gleichzeitig wieder zum Ausgangszustand $\psi\left(x_{1}, x_{2}\right)$ zurückgelangt. Folglich kann der Phasenfaktor, der bei einer Vertauschung auftritt, nur \pm 1 betragen. Diese beiden Möglichkeiten entsprechen genau den beiden Möglichkeiten in drei Dimensionen. Für Bosonen gilt $\psi\left(x_{1}, x_{2}\right)=\psi\left(x_{2}, x_{1}\right)$. Für Fermionen ist dagegen $\psi\left(x_{1}, x_{2}\right)=-\psi\left(x_{2}, x_{1}\right)$. Daraus folgt sofort, daß sich zwei Fermionen nie gleichzeitig am selben Ort befinden dürfen, da man durch Vertauschen der beiden Fermionen die Bedingung $\psi\left(x_{1}, x_{1}\right)=-\psi\left(x_{1}\right.$, $x_{1}$ ) erhält, die für nichtverschwindende Wel- lenfunktionen nicht erfüllt werden kann. Allgemein gilt für Fermionen das Pauli-Prinzip. Es besagt, daß sich zwei Fermionen nie im gleichen Zustand befinden dürfen. Für Bosonen gibt es dagegen ein solches Prinzip nicht. Es dürfen sich also mehrere Bosonen im gleichen Zustand befinden.

Eröffnet die Beschränkung auf zwei Dimensionen auch beim Verhalten gleicher Teilchen bei Vertauschung neue Möglichkeiten? Zur Beantwortung dieser Frage müsen wir die Vertauschung zweier Teilchen etwas genauer betrachten. Zwei Teilchen können in zwei Dimensionen miteinander vertauscht werden, indem man, wie in Abbildung 2 gezeigt, das eine Teilchen im Halbkreis um das andere Teilchen herumführt. Nach einer anschließenden unwichtigen Verschiebung beider Teilchen haben wir tatsächlich die gewünschte Vertauschung erzielt. Nach einer weiteren Vertauschung dieser Art haben die Teilchen wieder ihre ursprünglichen Plätze eingenommen. Der Anfangszustand ist jetzt jedoch sehr wohl vom Endzustand zu unterscheiden. Dies läßt sich leicht nachvollziehen, wenn wir zu unserem Tischtennisball-Modell zurückkehren und diese Vertauschungsoperationen mit je einem Ball in jeder Hand nachvollziehen. Die dabei entstehende „Verknotung" der Arme zeigt deutlich den Unterschied zwischen Anfangs- und Endzustand. Ausgehend von einem bestimmten Anfangszustand kann man nun dem Endzustand eine sogenannte Windungszahl zuordnen, die angibt, wie oft und in welche Richtung das eine Teilchen um das andere Teilchen herumgeführt wurde. Die Windungszahl ist für den Weg des Teilchens charakteristisch. Hält man nämlich Anfangs- und Endpunkt fest, so läßt sich ein Weg mit einer bestimmten Windungszahl nicht durch kontinuierliches Verformen in einen Weg mit einer anderen Windungszahl überführen. Dies gilt jedoch nur in zwei $\mathrm{Di}^{-}$ mensionen. In drei Dimensionen läßt sich jeder geschlossene Weg unter Umgehung des anderen Teilchens zu einem Punkt zusammenziehen. Für die verknoteten Arme bedeutet das, daß wir sie unter Ausnutzung der dritten Dimension sofort wieder entknoten können (was der Leser, falls er das kleine Experiment selbst durchgeführt hat, spätestens jetzt auch tun sollte). Dies ist letztendlich auch der Grund, weswegen wir oben gesagt haben, daß ein Zustand und der durch zweimaliges Vertauschen entstandene Zustand in drei Dimensionen durch die gleiche Wellenfunktion beschrieben werden müssen. Der durch die Vertauschung beschriebene Weg 
läßt sich nämlich in einen Weg verformen, bei dem überhaupt keine Vertauschung stattgefunden hat.

Wir merken noch an, daß sich die Windungszahl auch in zwei Dimensionen unter Umständen austricksen läßt. Können sich die beiden Teilchen nämlich gegenseitig durchdringen, so lassen sich Wege auch in zwei Dimensionen immer zusammenziehen. In diesem Fall kann es nur Bosonen geben, da sich Fermionen, wie wir bereits wissen, nie am selben Ort befinden können und sich daher auch nicht durchdringen können.

Da sich in zwei Dimensionen, wenn man von dem trivialen Fall sich durchdringender Teilchen absieht, ein Zustand und der daraus durch zweimaliges Vertauschen der Teilchen hervorgehende Zustand unterscheiden lassen, müssen zwar die jeweiligen Ortsaufenthaltswahrscheinlichkeiten übereinstimmen, nicht aber die Wellenfunktionen. Letztere dürfen vielmehr um einen Phasenfaktor $\exp (\mathrm{i} 2 \pi v)$ verschieden sein. Das einmalige Vertauschen zweier gleicher Teilchen liefert also einen Faktor $\exp (i \pi v)$. Darin sind die Bosonen mit $v=O$ (Phasenfaktor +1$)$ und die Fermionen mit $v=1$ (Phasenfaktor -1 ) enthalten. Dazwischen liegende Werte von $v$ entsprechen „irgendwelchen Teilchen", eben Anyonen. Sie stellen gewissermaßen eine Verbindung zwischen Bosonen und Fermionen her, wie wir auch am Beispiel des zweidimensionalen Oszillators sehen können (siehe „Zwei harmonisch gekoppelte Anyonen“, Seite 85).

\section{Weltlinien und die Zopfgruppe}

Die Bewegung von Anyonen läßt sich mit Hilfe ihrer Weltlinien in einem dreidimensionalen Raum darstellen. Dabei benötigt man zwei Dimensionen für den Raum in dem die Anyonen leben, und die dritte Dimension hat die Bedeutung der Zeit. Wir wollen zunächst noch einmal die Vertauschung zweier Anyonen betrachten, die in Abbildung 3 dargestellt ist. Das eine Anyon wird an seinem Ort festgehalten, womit sich als Weltlinie eine Gerade in Zeitrichtung ergibt. Das andere Anyon wird, wie im letzten Abschnitt diskutiert, um das erste Anyon herumgeführt. Man erkennt deutlich die Windung, die sich durch zweimaliges Vertauschen der Anyonen ergibt. Hält man Anfangs- und Endpunkte der Weltlinien fest, so ist es nicht möglich, die gewundene Weltlinie in eine Gerade zu verformen. Dies zeigt nochmals die Existenz von Windungszahlen in zwei Dimensionen.

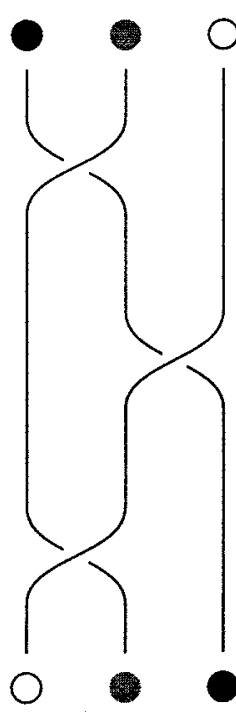

a

Abb. 4. Zwei äquivalente Vertauschungssequenzen im zweidimensionalen Raum, die einen wesentlichen Bestandteil der Definition der Zopfgruppe bilden.

Im nächsten Schritt betrachten wir die Vertauschung von drei Anyonen. In Abbildung 4a werden zunächst die beiden linken Anyonen wie oben diskutiert miteinander vertauscht. Anschließend sind die beiden rechten Anyonen an der Reihe und schließlich wieder die beiden linken Anyonen. Auf diese Weise haben die beiden Anyonen, die sich ursprünglich links und rechts befanden, ihre Plätze getauscht, während das mittlere Anyon seinen Platz beibehalten hat. Der Übergang zwischen dieser Anfangs- und Endstellung der Anyonen läßt sich aber auch anders bewerkstelligen, wie in Abbildung 4b $\mathrm{zu}$ sehen ist. Dabei werden zunächst die beiden rechten Anyonen miteinander vertauscht, dann die beiden linken und abschließend wieder die beiden rechten Anyonen.

Sind die beiden Vertauschungssequenzen der Abbildung 4 äquivalent, mit anderen Worten, ändert sich die Wellenfunktion der drei Anyonen um denselben Phasenfaktor? Um dies zu entscheiden, müssen wir nur die Anfangs- und Endpunkte der Weltlinien festhalten und versuchen, die Weltlinien der Abbildung $4 \mathrm{a}$ in die Weltlinien der Abbildung $4 \mathrm{~b}$ zu überführen. Dies ist in der Tat möglich. Davon kann man sich durch Überlegen oder „experimentell“ mit Hilfe von Schnüren überzeugen.

Die Äquivalenz der Vertauschungen in den Abbildungen $4 \mathrm{a}$ und $4 \mathrm{~b}$ zusammen mit der Tatsache, daß Verdrillungen in verschiedenen Paaren von Weltlinien unabhängig voneinan- der sind, definieren die sogenannte Zopfgruppe, die ihren Namen von den zopfartigen Gebilden hat, die bei den Verdrillungen entstehen. Diese Gruppe bildet die Grundlage zur mathematischen Beschreibung von Anyonen. Die Zopfgruppe ist übrigens ein ziemlich alter Zopf. Sie wurde nämlich schon in den zwanziger Jahren dieses Jahrhunderts von dem Mathematiker Emil Artin in Hamburg studiert.

\section{Verletzung der Invarianz unter Spiegelung und Zeitumkehr}

Wir wollen die mathematische Seite der Anyonen hier nicht weiter vertiefen, sondern uns jetzt mehr der Physik zuwenden. Dabei ist es immer wichtig, die Symmetrien eines Systems kennenzulernen. Man kann beispielsweise fragen, ob sich ein bestimmter Vorgang von seinem Spiegelbild unterscheiden läßt. Ist dies der Fall, so sagt man, die Parität sei verletzt. Eine andere Frage ist die nach der Zeitumkehrinvarianz. Dazu stellt man sich vor, man habe einen physikalischen Vorgang gefilmt und spiele den Film jetzt rückwärts ab. Lassen sich die beiden Vorgänge nicht voneinander unterscheiden, so ist das System invariant unter Zeitumkehr.

Zur Veranschaulichung betrachten wir zunächst ein Elektron, das sich senkrecht auf eine negativ geladene Metallplatte zubewegt. Durch die elektrostatische Abstoßung wird das Elektron abgebremst, kommt schließlich zum Stillstand und dreht seine Bewegungsrichtung um. Offensichtlich sieht der Vorgang, im Spiegel betrachtet, genauso aus. Auch die Zeitumkehrinvarianz ist erfüllt. Läßt man den ganzen Vorgang nämlich rückwärts laufen, so erhält man genau den gleichen Ablauf wieder.

Als zweites Beispiel betrachten wir die Bewe gung eines Elektrons in einem Magnetfeld. Die Kreisbahn muß bei der in Abbildung 5a gewählten Orientierung des Magnetfeldes immer im gezeigten Umlaufsinn durchlaufen werden. Betrachten wir die Situation im Spiegel, so erhalten wir die Abbildung 5b. Dabei hat sich der Umlaufsinn umgekehrt. Dieser Vorgang ist physikalisch nicht erlaubt, wir haben also eine Paritätsverletzung vorliegen. Die Zeitumkehrinvarianz ist ebenso verletzt, $\mathrm{da}$ auch hier der Drehsinn umgekehrt wird.

Wie verhält sich nun die Vertauschung von Anyonen unter Spiegelung und Zeitumkehr? Aus Abbildung 6 erkennt man sofort, $\mathrm{da}$ die 
Vertauschung zweier Anyonen nicht invariant unter Spiegelung und Zeitumkehr ist. Betrachten wir nämlich die Situation im Spiegel, so stellen wir fest, daß das bewegte Anyon genau in der umgekehrten Richtung transportiert wird, der Drehwinkel beträgt statt $180^{\circ}$ gerade $-180^{\circ}$. Damit treten die Phasenfaktoren $e^{i \pi v}$ und $e^{-i \pi v}$ auf, die verschieden sind, sofern es sich bei den Teilchen nicht um Bosonen oder Fermionen mit $v=0$ beziehungsweise $v=1$ handelt. Entsprechendes gilt auch für die Zeitumkehr, da die rechte Vertauschung als zeitumgekehrte Version der linken Vertauschung verstanden werden kann. Die Verletzung der Invarianz unter Parität und Zeitumkehr ist von Bedeutung, wenn man sich auf die experimentelle Suche nach Anyonen macht. Ist nicht ohnehin ein Magnetfeld vorhanden, so deutet die Verletzung dieser Invarianzen auf eine mögliche Anwesenheit von Anyonen hin, auch wenn dies als Beweis natürlich nicht ausreicht.

\section{Aharonov-Bohm-Effekt}

Wir wollen jetzt versuchen, ein etwas anschaulicheres Bild der Anyonen zu gewinnen. Im letzten Abschnitt haben wir gesehen, daß sowohl bei der Bewegung einer Ladung im Magnetfeld als auch bei der Vertauschung zweier Anyonen die Parität und die Zeitumkehrinvarianz verletzt sind. Diese Parallelität läßt sich ausnutzen. In der Tat ist die Einführung eines fiktiven magnetischen Flusses zum Verständnis von Anyonen recht hilfreich.

Wir schweifen zunächst etwas ab und diskutieren den nach Yakir Aharonov und David Bohm benannten Effekt, der mit dem Transport einer Ladung um ein räumlich lokalisiertes Magnetfeld zusammenhängt. Die Situation ist in Abbildung 7 genauer dargestellt. In der Mitte befindet sich in einem dünnen, schlauchförmigen Bereich ein Magnetfeld $\vec{B}$, das über die Fläche integriert einen magnetischen Fluß $\Phi$ ergibt. Es ist praktisch, außerdem das Vektorpotential $\vec{A}$ einzuführen, dessen Wirbel das Magnetfeld bestimmt. Während das Magnetfeld lokalisiert ist, hat das Vektorpotential überall einen endlichen Wert und fällt nur bei sehr großen Abständen vom Wirbelmittelpunkt gegen Null ab. Klassisch hat das Vektorpotential keinerlei Bedeutung. Ein geladenes Teilchen, das sich wie in Abbildung 7 außerhaib des Magnetfeldes bewegt, erfährt keine Kraft durch das Magnetfeld. Es weiß also nichts von dem magnetischen Fluß, den es umrundet.
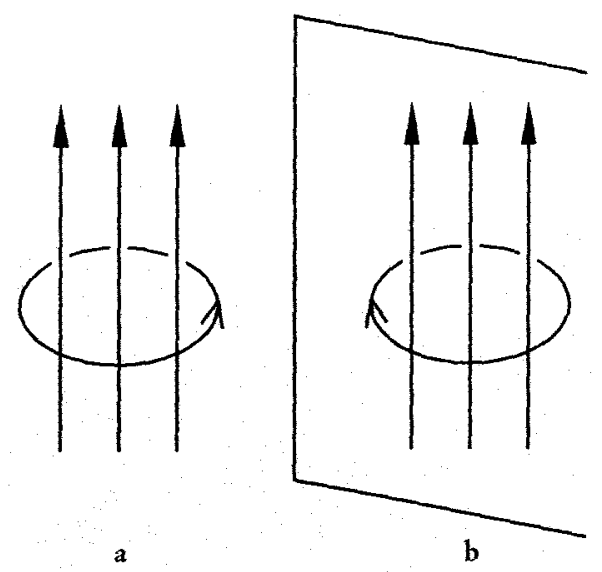

Abb. 5. Bewegung eines Elektrons im Magnetfeld (a) und die physikalisch nicht mögliche, gespiegelte oder zeitumgekehrte Version (b).

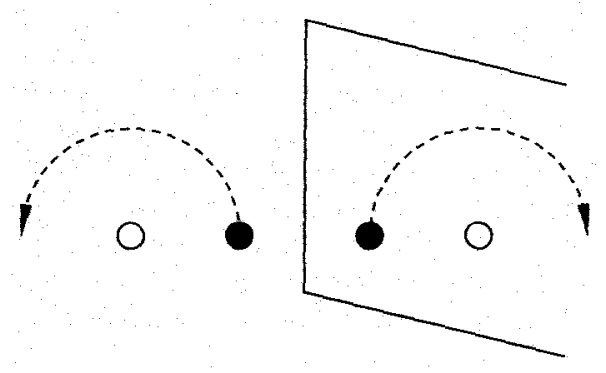

Abb. 6. Vertauschung zweier Teilchen und die gespiegelte oder zeitumgekehrte Version.

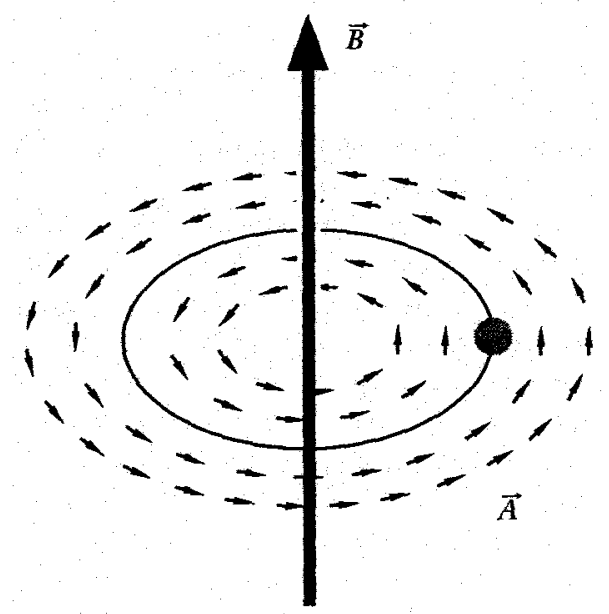

Abb. 7. Aharonov-Bohm-Effekt. Ein geladenes Teilchen wird um ein schlauchförmiges Magnetfeld transportiert. Die Phase der Wellenfunktion ändert sich aufgrund des Vektorpotentials.

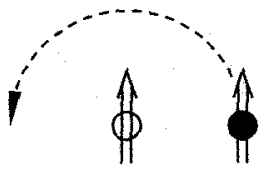

Abb. 8. Vertauschung von zwei Bosonen mit aufgepfropftem magnetischem Fluß.

Ein quantenmechanisches Teilchen dagegen interessiert sich nicht nur für das Magnetfeld, sondern auch für das Vektorpotential. Es stellt sich heraus, daß sich die Wellenfunktion des Teilchens bei einem vollen Umlauf um einen Phasenfaktor $\exp \left(-\mathrm{i} 2 \pi \Phi / \Phi_{0}\right)$ ändert, wobei $\Phi$ der umrundete magnetische Fluß und $\Phi_{0}=$ $h / q$ das Flußquant ist. Letzteres ist durch die Plancksche Konstante $h$ und die Ladung $q$ des umlaufenden Teilchens bestimmt. Diese Phasenänderung läßt sich nachweisen, wenn man zwei Teilchen in entgegengesetzter Richtung auf die Reise um das Magnetfeld schickt und bei ihrem Zusammentreffen auf der gegenüberliegenden Seite die Phasen der beiden Wellenfunktionen, die anfänglich gleich waren, miteinander vergleicht. Man beobachtet dann als Folge der verschiedenen Phasenänderungen ein Interferenzmuster in Abhängigkeit vom magnetischen Fluß.

Die Änderung der Phase beim Umlauf eines geladenen Teilchens um einen magnetischen Fluß erinnert uns natürlich stark an das Verhalten der Wellenfunktion zweier Anyonen, die umeinander herum laufen. Wenn wir von Bosonen ausgehen, deren Wellenfunktion sich bei Vertauschung ja nicht verändert, können wir uns daher ein Modell für Anyonen basteln. Wir geben dabei jedem Boson eine fiktive Ladung $q$ und einen fiktiven magnetischen Fluß $\Phi$, so daß gerade $\Phi / \Phi_{0}=v$ gilt. Zur Erinnerung, $v$ war die Größe, die das Verhalten der Anyonen unter Vertauschung charakterisiert. Dieses Bild ist für die Untersuchung von Anyonen häufig sehr nützlich, da man zunächst einfach nur mit Bosonen rechnen kann, die kein kompliziertes Verhalten bei Vertauschung aufweisen (siehe „Zwei harmonisch gekoppelte Anyonen“). Man sollte jedoch bedenken, daß der magnetische Fluß, den man dann zusätzlich berücksichtigen muß, eigentlich nicht real ist.

\section{Veränderung der Statistik}

Wir wissen bereits, daß die Vertauschungsregeln für Fermionen verhindern, daß zwei Fermionen gleichzeitig denselben Zustand besetzen, während dies für Bosonen erlaubt ist. Versuchen wir nun beispielsweise, zwei Zustände mit zwei Teilchen zu besetzen. Für 


\section{Zwei harmonisch gekoppelte Anyonen}

Einer Arbeit der Norweger Leinaas und Myrheim [4] folgend, betrachten wir zwei harmonisch gekoppelte Anyonen. Vernachlässigt man die Schwerpunktsbewegung, so läßt sich das Problem auf den zweidimensionalen harmonischen Oszillator mit dem Hamiltonoperator

$H=\frac{1}{2 m}\left(p_{x}^{2}+p_{y}^{2}\right)+\frac{m}{2} \omega^{2}\left(x^{2}+y^{2}\right)$

abbilden. Da die Anteile in $x$ - und $y$-Richtung separieren, ist es sinnvoll, sich zunächst die wichtigsten Resultate des eindimensionalen harmonischen Oszillators in Erinnerung zu rufen. Die Energieeigenwerte sind durch $E_{n}=(n+1 / 2) \hbar \omega, n=0,1,2, \ldots$ gegeben, wobei die zugehörige Eigenfunktion $\psi_{n}(x)$ für gerade $n$ symmetrisch und für ungerade $n$ antisymmetrisch ist. Die Eigenfunktion $\psi_{n m}(x, y)$ für den zweidimensionalen Fall läßt sich dann als Produkt $\psi_{n}(x) \psi_{m}(y)$ schreiben, wobei der zugehörige Energieeigenwert gleich $(n+m+1) b \omega$ ist.

Wir setzen nun zwei Bosonen in das zweidimensionale harmonische Potential und halten dabei den Schwerpunkt der Bosonen im Ursprung fest. Dann muß aufgrund der Vertauschungsregeln $\psi(x, y)=\psi(-x,-y)$ gelten. $\psi_{n m}(x, y)$ muß also als Produkt zweier symmetrischer oder zweier antisymmetrischer Eigenfunktionen des eindimensionalen $\mathrm{O}_{5}$ zillators geschrieben werden, also $(n, m)=$ $(0,0),(0,2),(1,1),(2,0), \ldots$ mit den Energieeigenwerten $\hbar \omega, 3 \hbar \omega$. . Für Fermionen muß dagegen $\psi(x, y)=-\psi(-x,-y)$ gelten. Es muß also jeweils eine symmetrische und eine antisymmetrische Wellenfunktion des eindimensionalen Oszillators miteinander multipliziert werden. Man erhält so $(n, m)=$ $(0,1) ;(1,0),(0,3) ;(1,2) ;(2,1),(3,0), \ldots . \mathrm{mit}$ den Energieeigenwerten $2 \hbar \omega, 4 \hbar \omega$.

Für Anyonen lassen sich die Vertauschungseigenschaften nicht mehr auf diese Art beruicksichtigen, Es ist vielmehr praktisch, zu Polarkoordinaten überzugehen, wobei dann $\psi(r, \varphi+\pi)=e^{i \pi} \psi(r, \varphi)$ gelten muB.

\section{Komplexe Zahlen}

Mit Hilfe der reellen Zahlen, die man gewöhnlich zum Rechnen verwendet, ist es nicht möglich, die Wurzel aus einer negativen Zahl zu ziehen. Um dieses Problem zu beseitigen, definiert man die sogenannte imaginäre Einheit 1 , die der Bedingung $i^{2}=$ -1 genügt. Mit Hilfe zweier reeller Zahlen $x$ und $y$ und der imaginaren Einheit kann man komplexe Zahlen bauen, indem man $z=x+$ iy setzt. Man bezeichnet $x$ als den Realteil und $y$ als den Imaginarteil. Mit diesen Zahlen kann man wie gewohnt rechnen, wenn man iy als Produkt zweier Zahlen auffaßt und $\mathrm{i}^{2}=-1$ berucksichtigt. Die zu z konjugiert komplexe Zahl $z$ definiert man als $\bar{z}=$ $x-y$, der Imaginarteil andert also sein Vorzeichen. Nach den Rechenregeln ergibt sich dann beispielsweise für das Betragsquadrat $|z|^{2}=z z$ einer komplexen $Z a h l|z|^{2}=x^{2}+y^{2}$. Komplexe Zahlen lassen sich wie in Abbildung 1 als Punkte in der $x-y$-Ebene veranschaulichen. Dabei kann man die komplexe
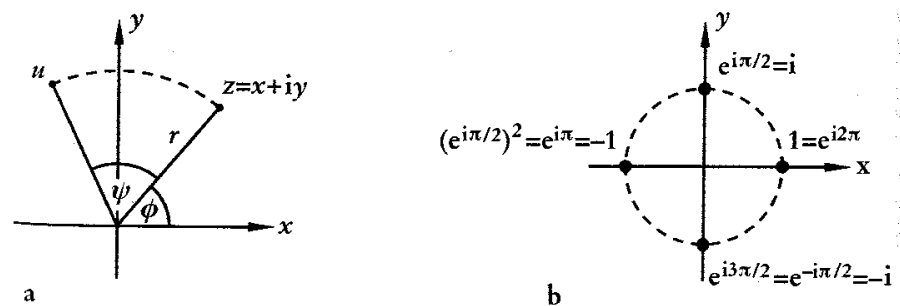

Abb. 1. (a) Die komplexe Zahlenebene. (b) Spezielle komplexe Zahlen mit Betrag Eins.
Diese Bedingung ist automatisch berucksichtigt, wenn man eine Funktion $\widetilde{\psi}(r, \varphi)=$ $e^{-\mathrm{i} v \varphi} \psi(r, \varphi)$ einführt und fordert, daß die neue Funktion $\pi$-periodisch in $\varphi$ ist. Die Wellenfunktion $\widetilde{\psi}(r, \varphi)$ genügt also den Vertauschungsregeln für Bosonen. Für die Schrödingergleichung ergibt sich dann

$$
\begin{aligned}
& {\left[-\frac{\hbar^{2}}{2 m}\left(\frac{\partial^{2}}{\partial r^{2}}+\frac{1}{r} \frac{\partial}{\partial r}+\frac{1}{r^{2}}\left(\frac{\partial}{\partial \varphi}+i v\right)^{2}\right.\right.} \\
& \left.+\frac{m}{2} w^{2} r^{2}\right] \tilde{\psi}(r, \varphi)=E \tilde{\psi}(r, \varphi),
\end{aligned}
$$

wobei der Anyonencharakter der beiden Teilchen jetzt in Form eines fiktiven Vektorpotentials im zusätzlichen Term i $v$ in der kinetischen Energie berücksichtigt ist. Man kann nun die Eigenenergie des zweidimensionalen harmonischen Oszillators direkt übernehmen, wenn man aufgrund des Vektorpotentials den Drehimpuls um v/2 verschiebt. Es ergibt sich somit

$$
E_{n l}=2 \hbar \omega\left(n+\left|l+\frac{v}{2}\right|+\frac{1}{2}\right), n, l=0,1,2, \ldots
$$

was in Einklang mit unseren obigen Überlegungen für Bosonen und Fermionen steht. Abbildung 2 zeigt die untersten Energieniveaus und deren Entartung für Bosonen, Anyonen mit $v=1 / 2$ und Fermionen. Man sieht deutlich, wie Anyonen eine Brücke zwischen Bosonen und Fermionen schlagen.

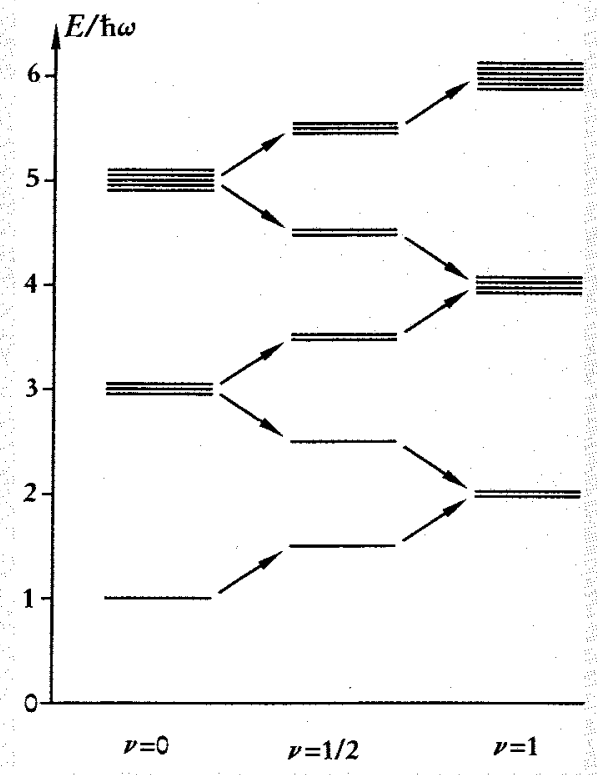

Abb. 2. Eigenenergien und Entartung für zwei harmonisch gekoppelte Teilchen (v. 1. n. r. Bosonen, Anyonen mit $v=1 / 2$ und Fermionen). 
Fermionen gibt es nur eine Möglichkeit, nämlich jeden Zustand mit einem Teilchen zu besetzen. Dabei führt das Vertauschen zu keiner zusätzlichen Möglichkeit, da die Teilchen ununterscheidbar sind. Für Bosonen gibt es dagegen drei verschiedene Möglichkeiten. Dieses Beispiel läßt sich natürlich auf kompliziertere und physikalisch interessantere Situationen verallgemeinern, wobei sich Bosonen und Fermionen immer verschieden verhalten. Man sagt deshalb, die beiden Teilchensorten gehorchen verschiedener Statistik.

In drei Dimensionen ist es möglich, aus einer geraden Anzahl von Fermionen Bosonen zu machen, wenn man nicht $\mathrm{zu}$ genau hinsieht. Bei Vertauschung zweier Fermionen erhält man einen Faktor $(-1)^{2}=+1$, der für Bosonen typisch ist. Supraleitung wird erst möglich, weil sich zwei Elektronen zu einem Cooperpaar zusammentun, das sich in gewisser Weise wie ein Boson verhält. Auch die Supraflüssigkeit des ${ }^{4} \mathrm{He}$, das aus zwei Protonen und zwei Neutronen, also vier Fermionen, besteht, kommt dadurch zustande, daß sich ${ }^{4} \mathrm{He}$-Kerne in diesem Fall wie Bosonen verhalten.

In zwei Dimensionen kann man nun die Statistik durch Aufpfropfen eines magnetischen Flusses wie im vorigen Abschnitt beliebig verändern. Man kann sogar aus Bosonen Fermionen machen, was in drei Dimensionen nach obigem Schema nicht möglich ist. Dazu versehen wir die Bosonen jeweils mit einem halben Flußquant. Die Vertauschung dieser Teilchen überlegen wir uns mit Hilfe von $\mathrm{Ab}$ bildung 8 . Das schwarze Teilchen erhält dadurch, daß es sich im Vektorpotential des weißen Teilchens bewegt, einen AharonovBohm-Phasenfaktor $e^{-\mathrm{i} \pi / 2}$. Dasselbe gilt aber auch für das weiße Teilchen, da es sich vom schwarzen Teilchen aus gesehen in dessen Vektorpotential bewegt. Das Gesamtsystem ändert seinen Phasenfaktor also um $\left(e^{-i \pi / 2}\right)^{2}=$ -1 . Bosonen mit einem aufgepfropften halben Flußquant verhalten sich also tatsächlich wie Fermionen. Gemäß der Diskussion im vorhergehenden Abschnitt können wir durch Variation des aufgepfropften Flusses beliebige Anyonen erzeugen. Wir können die Statistik also kontinuierlich verändern. Anyonen interpolieren zwischen bosonischer und fermionischer Statistik.

\section{Anwendungen}

Bis jetzt waren alle Überlegungen, die wir angestellt haben, theoretischer Natur, und wir müssen uns fragen, ob Anyonen etwas mit der Realität zu tun haben. Reale Teilchen leben immer in drei Dimensionen und können daher nur Bosonen oder Fermionen sein. Einen Ausweg bilden Anregungen in zweidimensionalen Systemen, also Systemen, in denen die dritte Dimension vernachlässigt werden kann. Solchen Anregungen können teilchenartige Eigenschaften zugeordnet werden, man spricht auch von Quasiteilchen. Gibt es also Quasiteilchen, die sich wie Anyonen verhalten?

In den letzten Jahren wurden zwei Systeme besonders intensiv auf ihren möglichen $\mathrm{Zu}$ sammenhang mit Anyonen hin untersucht. Ein System waren die neuen Hochtemperatursupraleiter, von denen man weiß, daß die Supraleitung in bestimmten Kristallebenen auftritt. Die notwendige Zweidimensionalität ist also vorhanden. Mit Hilfe optischer Experimente wurde versucht, die für Anyonen erwartete Symmetriebrechung von Parität und Zeitumkehr nachzuweisen. Nach anfänglich ermutigenden Ergebnissen ist eine Symmetriebrechung bis heute aber nicht eindeutig nachgewiesen worden [7]. Man muß vielmehr davon ausgehen, $\mathrm{da} ß$ zumindest in den heute studierten Hochtemperatursupraleitern Anyonen nicht für die Supraleitung verantwortlich sind.

Ein anderes System, in dem Anyonen eine Rolle spielen könnten, ist das zweidimensionale Elektronengas im Magnetfeld. Eine in Halbleiterheterostrukturen erzeugte dünne zweidimensionale Ansammlung von Elektronen zeigt bei bestimmten Werten des Magnetfeldes Plateaus in der Hall-Leitfähigkeit, den sogenannten fraktionellen Quanten-Hall-Effekt. Für eine genauere Diskussion verweisen wir auf zukünftige Artikel in dieser Zeitschrift. An dieser Stelle sei erwähnt, daß man die ausgezeichneten Werte des Magnetfeldes, für die Plateaus auftreten, in einer Hierarchie anordnen kann, und daß es Ansätze gibt, die diese Hierarchie mit Hilfe von anyonischen Anregungen zu erklären versuchen (siehe zum Beispiel [3], Kapitel 8). Es ist jedoch noch nicht klar, ob Anyonen wirklich die Lösung des Rätsels des fraktionellen Quanten-Hall-Effekts sein werden. Es bleibt also abzuwarten, ob Frank Wilczek recht hat, wenn er mit Blick auf die Anyonen sagt, daß die Natur Möglichkeiten, die sie hat, auch realisiert [1].

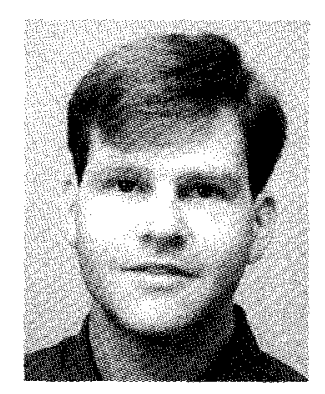

Gert-Ludwig Ingold, geboren 1960 in Stuttgart. Physikstudium an der Universität Stuttgart, Diplom 1985. 1985/86 an der State University of New York at Stony Brook, anschließend wieder an der Universität Stuttgart, Promotion 1988. Anschließend wissenschaftlicher Angestellter an der Universität-GH Essen, Habilitation 1993, seit 1994 Heisenberg-Stipendiat.

Anschrift:

Dr. Gert-Ludwig Ingold, Fachbereich Physik, Universität-GH Essen, D-45117 Essen. 